à laquelle on avait ajouté un indicateur et qui fut ensuite lavé au moyen d'eau contenant une substance alcaline ou acide.

$\mathrm{Si}$; dans un beurre fait de crème acidulée on introduit des sels alcalins, les gouttelettes du liquide seront, d'après TUOMAINEN, pour le moins, partiellement neutralisées.

En rapport avec le procédé finlandais de fabrication de beurre stable, dans lequel des sels alcalins sont introduits dans le beurre durant le malaxage, l'examen susdit a une grande importance. Mais, Tuomainen ne se prononce pas au sujet d'une neutralisation qui pourrait être due à une diffusion de contenu des gouttelettes acides vers les gouttelettes alcalines.

Des résultats des expériences sur la diffusion, Tuomainen, déduisait que : il peut se produire dans le beurre un "courant" de liquides, peut être à travers les membranes superficielles des globules de graisse; peut être d'une autre manière. Cette diffusion se laisse bien harmoniser avec la théorie sur la structure du beurre de Mulder, citée ci-dessus.

\title{
BIBLIOGRAPHIE
}

[1] H. Mulder. Zuivelonderzoeh deel I en II, ed. Alg. Ned Zuivelbond, Den Haag.

[2] M. G. Ter Horst. Neth. Milk et Dairy Journal, 1, 137, 1947.

[3] H. Murdwr. Versl. landbk. onderz.,'51, 467, 1945.

\section{LA COMPOSITION CHIMIQUE DU LAIT ET DES PRODUITS LAITIERS}

\author{
Rapport général présenté \\ par
}

\section{J. KEILLING}

Ing. Agr., Directeur du Laboratoire des Industries Laitières, Sèvres (France)

\section{INTRODUCTION}

Sous le titre : "La composition chimique du lait et des produits laitiers " ont été groupé 25 rapports, dont la elassification en vue d'une présentation d'ensemble n'a pas été la moindre tâche du rapporteur général. En effet, l'on peut dire que chacun de ces rapports aborde un aspect différent de l'évolution de nos connaissances d'ordre chimique ou similaires dans le lait et les industries laitières, quelles qu'elles soient.

Pendant que certains rapports sont constitués par une documentation analytique qu'il convient de verser aux archives chimiques de nos industries, dont ils constituent un complément important; 
d'autres commentent des procédés nouveaux appliqués dans les diverses professions,

d'autres enfin apportent soit données nouvelles, soit techniques analytiques nouvelles.

- Aussi le premier travail fut-il de choisir un mode de classification qui permit d'embrasser l'ensemble des textes donnés, sous cette rubrique, au Congrès de 1949 :

1. Données analytiques complémentaires.

2. Application des techniques nouvelles à la chimie laitière.

3. Technique industrielle nouvelle.

\section{Données analytiques complémentaires}

A. L. Provan. Investigations into the composition of milk.

E. Lauprecht et H. Dörtng. Uber die Einflüsse von Jahresmilehmenge, Alter und Lebendgewicht der Kühe auf den Fettgehalt der Milch.

S. Y. Thompson, J. Ganguly, E. H. Mawson et S. K. Kon. Studies of vitamin $A$ in milk in Great Britain.

V. MAXA. The decreases of vitamin C during some treatments of milk.

F. H. MoDowall. The properties of New Zealand butterfat.

P. JАмотпе. Les teneurs en fer et en euivre de quelques beurres belges.

T. STORGARDs. The protein content in butter.

J. Csiszar et A. Bakos. The lecithin content of the buttermilk-cheese.

A. ZeILINGER. Auswirkungen des Zusatzes von Lab bei der Herstellung von Speise-Topfen aus saurer Buttermileh.

J. Csiszar. Correlation between the water content and quality of the "Ovar" cheese.

H. Torssell, U. SAnd berg et L. E. Thureson. Changes in viscosity and conductivity during concentration of milk.

J. Ferrer. Modifications chimiques du beurre, durcissement par hydrogénation.

Sur les composants du lait Provan rapporte et commente les résultats obtenus dans les laboratoires laitiers d'Angleterre et d'Ecosse.

Il met en relief la variation progressive de la composition chimique des nombreux laits examinés et notamment la réduction des extraits secs non gras qui passent de $8,91 \%$ en 1933 à 8,6\% en 1946, pour un ensemble de 5.800 producteurs tandis que la matière grasse déclinait beaucoup moins nettement, de $3,6 \%$ à l'origine à $3,5 \%$ actuellement.

De cette étude qu'il serait intéressant de rapprocher d'un récent travail d'EYRARD pour les laits français, aucune conclusion pratique ne peut être tirée, quant aux causes profondes de cette variation de richesse, dont la répercussion sur la valeur industrielle du lait est loin d'être négligeable. 
LAUPRECHT et DöRING étudient l'influence de la production annuelle, de $l^{h} \hat{a g g}$ et du poids des vaches sur la teneur en matière grasse du lait. Ils font intervenir deux constantes $a$ et $b$, caractéristiques des troupeaux observés dans la formule :

$z=\frac{a}{x}+b$

$z$ étant la teneur en matière grasse

$x$ la quantité de lait.

Ils observent ensuite les modifications de $a$ et de $b$, sur des troupeaux importants, en fonction de la teneur en matière grasse, de l'âge et du poids deś animaux.

A l'accroissement des quantités annuelles de lait correspond une augmentation de $a$, tandis que $b$ ne présente pas de variation caractéristique. Pour l'influence de l'âge des animaux, $b$ reste sensiblement constante tandis que $a$ passe par un maximum entre 5 et 7 ans.

Enfin l'accroissement du poids des animaux provoque un accroissement de $b$, tandis que les variations de $a$ semblent n'avoir aucune relation avec celles du poids des animaux.

Les vitamines nous ont valu deux rapports : dans le premier Thompson, Ganguly, Mawson et Kon ont étudié à la fois la teneur en vitamine A des laits en Grande-Bretagne et certaines particularités de cette vitamine : le carotène actif représente de 60 à $80 \%$ des caroténoïdes totaux, sur des échantillons moyens. Dans les laits individuels cette proportion peut varier de 25 à $90 \%$ : la vitamine A se trouve presque exclusivement sous la forme alcoolique dañs le lait.

$\mathrm{Au}$ cours des transformations du lait en beurre et fromages il est apparu que la teneur en vitamine A restait sensiblement la même dans toutes les fractions obtenues, tandis que celle des autres caroténoïdes variait en raison inverse de la dimension des globules gras.

Dans le second Maxa étudie le destin de la vitamine $\mathrm{C}$ dans les divers traitements du lait. Il souligne la nécessité de préserver cette vitamine le plus possible dans les industries laitières, où seule la fabrication du yaourt semble la préserver de façon convenable.

Seuls l'acier inoxydable, l'aluminium et l'étain respectent la vitamine C. L'influence de la coloration des bouteilles de verre est également soulignée.

Les conclusions de ce rapport sont conformes aux données classiques et représentent une bonne revue d'ensemble, aveo chiffres à 
l'appui, des pertes en vitamine $\mathrm{C}$ et des moyens de les réduire dans la pratique industrielle laitière.

Sur les problèmes strictement beurriers, les propriétés de la matière grasse des beurres néozélandais sont traitées par MCDowALL. Contrairement à ce qui se produit dans la plupart des autres pays laitiers l'indice d'iode des beurres néozélandais est le plus faible pendant la période d'abondance du pâturage et de forte production laitière.

Les variations d'indice d'iode sont très différentes dans les régions Nord et Sud de la Nouvelle-Zélande. Dans le Sud de ce pays la courbe de variation se rapproche des courbes de l'hémisphère Nord, où les conditions d'alimentation hivernale sont semblables.

Il n'y a pas de relation entre les changements de l'indice d'iode et le point de fusion des beurres : les points de fusion sont uniformément bas pendant la période de printemps et le début de l'été, ils s'élèvent pendangt l'automne et l'hiver.

Ces constatations mettent en évidence l'intérêt scientifique et technique certain d'études comparatives des matières grasses poursuivies sous différentes conditions de production. Leur confrontation éclairerait, à coup sûr, d'un jour nouveau, les études sur la physiologie des matières grasses.

Passant ensuite aux constatations ayant trait aux conditions de travail des beurres dans l'industrie nous rencontrons le rapport de JamotTe qui nous dacumente sur les teneurs en fer et cuivre des beurres belges.

Et le rapport de STORgards qui étudie la teneur des beurres en protéines. Indépendamment du rôle néfaste que peuvent jouer, au point de vue bactériologique, les protéines qui demeurent dans le beurre, un rôle utile leur est attribué : l'aetion antioxygène de protection de la matière grasse et c'est dans ce but qu'a été entreprise cette étude. Un net enrichissement des gouttelettes de babeurre retenues dans le beurre est mis en évidence, et il est en relation avec la dimension des grains de beurre. Plus ces derniers sont petits, plus le babeurre retenu est riche en protéine. Ce rapport ne mentionne pas l'état dans lequel se trouvent les protéines ainsi retenues.

Le babeurre nous a également valu deux études : l'une de CsISZÁr et BAKos sur la teneur en lécithine des fromages de babeurre confirme et précise les données classiques résultant de la supériorité biologique du babeurre sur le lait écrémé. Elle indique en outre une bien plus grande richesse en lécithines ( 3 fois) dans le fromage de babeurre que dans le fromage de lait entier. Le fromage de babeurre peut se ranger parmi les aliments riches en lécithines.

L'autre étude est de ZEILINGER et porte sur la modification de 
fabrication des produits alimentaires à partir du babeurre au moyen de présure.

La fromagerie a fait l'objet de deux rapports de Csiszár. Dans le premier est mise en évidence l'existence d'une relation définie entre la qualité des fromages et leur teneur en eau : la qualité variant, dans certaines limites, en raison inverse de la teneur en eau.

Cette constatation confirme ce que la plupart des industries fromagères européennes ont pu constater au cours de la période difficile qu'elles yiennent de traverser.

Il est regrettable qu'une étude analogue n'ait pas, à cette occasion, été entreprise sur la relation qui existe entre teneur en matière grasse et qualité, car il semble que ses conclusions eussent été davantage nuancées.

Dans le second travail d'ordre fromager, CsIszÁr rapporte ses études sur le changement du poids spécifique des sérums dans le but d'en distinguer l'origine. Aucun moyen simple n'a été déterminé qui permette de classer les sérums en fonction de leur origine.

Enfin, pour terminer ce groupe de communications, abordant un important problème industriel, Torsserl, SANDBERg et THURESON ont établi une étude sur la viscosité et la conductivité du lait au cours de sa concentration. Ils proposent une formule mathématique qui relie viscosité, température et l'extrait total dans le lait 'standardisé pour les fabrications et une autre formule pour le lait écrémé. Quant à la conductivité il n'a pas été possible de la relier par une formule à la température et à l'extrait sec total.

\section{Applications de technique nouvelles à la chimie laitière}

Nous retrouvons groupés sous cette rubrique la plupart des problèmes actuels de l'industrie laitière, examinés avec des techniques nouvelles ou suivant des préoccupations renouvelées.

1. L'étude de la constitution des protides du lait nous a valu deux intéressants rapports, utilisant l'un et l'autre des techniques relativement nouvelles, tout au moins dans leur utilisation dans ce domaine.

Hostettler et Rychener apportent de nouvelles données sur la caséine dans son état natif. Ils obtiennent, par ultracentrifugation, 5 fractions distinctes tant par leur aspect et leurs propriétés physiques que par leur composition chimique.

Pyne applique la polarographie à l'étude du complexe " phosphocaséinate de baryum ", et non pas comme l'indique le titre de son rapport, au phospho-caséinate de caloium. Cette technique fait apparaître la non identité des polarogrammes du caséinate de 
baryum et du phospho-caséinate de baryum. Par contre le complexe phospho-caséinate, après chauffage à $100^{\circ} \mathrm{C}$. pendant 30 minutes donne un polarogramme très voisin de celui du caséinate seul. Il semblerait donc que phosphate et caséinate sont, dans le complexe, unis par voie chimique, les acides aminés et le baryum intervenant dans la combinaison.

L'une et l'autre de ces communications ont le caractère de travaux préliminaires, susceptibles de développements ultérieurs.

2. Le non moins important problème de la valeur alimentảire du lait et des produits laitiers fait l'objet de deux communications extrêmement intéressantes tant au point de vue scientifique qu'au point de vue industriel.

HaRtman, Dryedn et Cary ont mis expérimentalement en évidence l'existence d'un facteur de croissance dans le lait et dans le lait écrémé, qu'ils identifient ou plutôt qu'ils apparentent à la vitamine B12 et qui donne les même résultats que l'extrait de foie utilisé pour le traitement de l'anémie pernicieuse. Ce facteur est présent dans les caséines commerciales, dans le lait, le lait écrémé et le lait sec, - son existence explique les résultats obtenus avec le lactose purifié cristallisé et avec les caséines purifiées et qui diffèrent essentiellement de ceux que l'on obtient avec le lait écrémé. C'est un argument de poids pour préciser l'incomparable valeur physiologique du lait.

JANSEN et GROOT revenant sur des travaux antérieurs concernant l'action favorable de l'acide vaccénique dans la nutrition et la croissance des jeunes rats, indiquent que cette action doit être attribuée non pas à cet acide mais à des impuretés qui l'accompagnent soit dans le beurre soit dans sa préparation au laboratoire.

Cette constatation les conduit à reprendre le problème de la valeur nutritive du beurre dans son ensemble, et à rapporter nombre d'indications qui ont trait à la différence de valeur biologique entre les beurres et les autres aliments gras.

Le moins que l'on puisse tirer de ce rapport et des faits qu'il cite est que le débat est loin d'être achevé et l'étude du problème loin d'être complète. Il faut souhaiter ici que cette entreprise fasse l'objet du travail de nombreux laboratoires, en raison même de l'extrême variété des beurres.

3. La chimie fromagère se préoccupe de trois questions à l'ordre du jour :

- l'arome des fromages, par analogie avec l'arome des beurres,

- les acides aminés des fromages dans leurs rapports avec la saveur de ceux-ci,

- le destin de la phosphatase et le test de la phosphatase dans es fromages. 
Calbert et Price ont recherché la présence et le rôle du diacétyle dans le fromage de Cheddar. Il leur est apparu que la classification des fromages suivant les goûts des experts n'avait aucun rapport avec leur teneur en diacétyle, mais que cependant la majorité des fromages classés comme excellents étaient très pauvres en diacétyle (moins de $0 \mathrm{mgr}$. 5 par kilogramme). Par contre, il n'est pas possible de rendre le diacétyle seul responsable des saveurs désagréables des mauvais fromages, mais bien plutôt d'autres produits de fermentation.

L'agitation du caillé pendant la préparation du fromage favorise la formation du diacétyle, ainsi que l'acidification pendant l'égouttage, tandis que, au cours de l'affinage, la teneur en diacétyle du fromage va en diminuant régulièrement.

Ce travail apporte de nouveaux éléments à l'étude des levains en fromagerie, où le problème semble devoir se poser différemment qu'il ne se pose pour la maturation des erèmes.

La dégradation de la caséine au cours de l'affinage a fait l'objet de deux études essentiellement distinctes quant aux méthodes analytiques utilisées.

HARPER et SWASON, travaillant sur le fromage de Cheddar et utilisant les techniques relativement nouvelles de dosage microbiologique des acides aminés rapportent qu'il existe un parallélisme, satisfaisant entre la teneur en acides aminés solubles dans l'eau et la saveur des fromages.

Storgards et Hietaranta utilisent, pour étudier la protéolyse au cours de l'affinage de l'Emmental les méthodes chimiques; ils constatent que le goût caractéristique de l'Emmental affiné est en rapport avec sa teneur en acide glutamique et aspartique, qui croît au cours de l'affinage. L'acide glutamique semble être le principal élément responsable de cette saveur.

Ces résultats sont à rapprocher de ceux du rapport de HARPER et Swanson, et des travaux de Meilder. Pour les uns etles autres, l'acide glutamique est mis en cause soit seul, soit accompagné d'autres acides aminés dans la saveur soit du Cheddar soit du Gouda. Cet acide aminé serait done facteur du goût de plusieurs fromages, et non pas caractéristique de l'Emmental. VIRTANeN, Kreula et NuRmikko ont recherché le rôle des acides aminés sur le goût de l'Emmental et la diminution de certains acides aminés au cours de la maturation des fromages.

Aussi devons-nous, plus particulièrement pour ce problème, souligner l'intérêt majeur d'une extension des études et recherches, tant sur le plan biologique que sur celui des modalités de fabrication. et d'affinage.

L'épreuve de la recherche de la phosphatase (phosphatase test) 
a été employée depuis déjà plusieurs années pour le contrôle de l'efficacité des traitements de pasteurisation dans le lait, la erème et le beurre. Les auteurs américains (SANDERS et SAGE) ont proposé l'extension de ce contrôle aux fromages élaborés à partir de lait pasteurisé, mais il ne semble pas, suivant le rapport que nous soumet CAMUS, que cette extension puisse se faire aisément car pour nombre de fromages la microflore superficielle, microbienne ou fongique, libère d'importantes quantités de phosphatases qui diffusent dans la pâte des fromages et peuvent ainsi mettre en échéc cette méthode de contrôle de pasteurisation. Il semblerait, d'après ce travail, que des études complémentares devraient être entreprises, tout au moins pour ce qui concerne les fromages affinés et leur microflore earactéristique eu égard à la généralisation de ce test.

4. La dessiccation des laits se heurte aux difficultés d'insolubilisation d'une part, de perte de valeur biologique d'autre part. Ces deux problèmes sont chacun l'objet d'une communication.

- L'une aborde la question de la composition des fractions solubles et insolubles des poudres de lait (WAITE et WHITE). Sur cet aspect primordial des séchages du lait, dont l'étude peut et doit orienter l'évolution des techniques industrielles il est confirmé que l'élément principal est la dénaturation des protéines au cours du traitement du lait. Une revue critique des différentes méthodes de détermination de la solubilité des laits en poudre montre la nécessité de l'élaboration de techniques nouvelles.

- L'autre rapporte les résultats des études de HENRY, KON, LEA et White sur la dégradation de la protéine dans le lait en poudre au cours du stockage.

Effectué sur des produits écrémés ce travail est valable pour le lait gras et porte sur les points suivants :

- l'humidité de $7,5 \%$ provoque transformation quasi totale du lactose de la forme $\beta$ en forme $\alpha$, au bout de 400 jours à $37^{\circ} \mathrm{C}$., tandis qu'à 3 et $5 \%$ d'humidité cette évolution est nulle.

L'humidité excessive accélère également la réduction du groupe des acides aminés libres, par réaction probable entre le lactose et les acides aminés dont le corollaire est un notable dégagement de $\mathrm{CO}^{2}$. Egalement, humidité élevée et température élevée agissent pour accélérer la perte de solubilité et la décoloration brune caractéristique du vieillissement des poudres de lait.

La dégradation des acides aminés porte notamment sur la lysine.

Les essais de détermination de la valeur biologique sur rats ont montré une perte considérable de cette valeur pour les poudres à haute humidité $(7,5 \%)$ stockées à haute température $\left(37^{\circ}\right.$ C.), mais non pour les poudres à $5 \%$ d'humidité. Cette perte était compensée par une addition de $1,25 \%$ de lysine. L'histidine à $0,5 \%$ 
éliminait la différence due au stockage à l'air comparé au stockage à l'azote.

Ce travail apporte une importante contribution à nos connaissances en cette matière et explique, pour une grande' part, nombre de difficultés survenues dans l'emploi des poudres de lait à l'issue des hostilités. Il présente également, à nos yeux, l'avantage d'orienter les techniques de fabrication de façon plus précise.

\section{Technique industrielle nouvelle}

Enfin, dans une eatégorie spéciale et faute d'avoir pu faire autrement nous examinerons un dernier rapport sur la Désacidification électrique du lait par CASALIS.

Traitant cette question à nouveau et après plusieurs auteurs plus anciens, il montre l'application intéressante du courant continu à faible voltage à la réduction de l'acidité des layits. L'emploi de ce procédé, avec un appareillage simple et nouveau permet de ramener à des acidités normales des laits déjà en voie de forte acidification, et rend d'énormes services dans les régions où les conditions naturelles et l'éducation insuffisante des producteurs de lait rendent inévitable l'apparition saisonnière d'une forte quantité de matière première inutilisable en l'état.

L'étude scientifique du phénomène est amorcée, non achevée, et l'application industrielle précède, comme dans beaucoup de cas, les études de laboratoire et leur trace fréquemment la voie.

\section{BIBLIOGRAPHIE ANALYTIQUE}

\section{$1 \circ$ LES LIVRES}

Die Schweizerische Milchwirtschaft (L'industrie laitière suisse), publié par la Cómmission Suisse du Lait, en collaboration avec des experts en matière d'industrie laitière. Un livre abondamment illustré et relié toile, 876 pages, format 18,5x26,5. Edité par Verlags-Aktiengesellschaft:Thun, 1948. Prix : 70 francs suisses.

Nous sommes très heureux de présenter cet ouvrage à nos leeteurs. I s'agit d'une véritable encyclopédie de l'industrie laitière suisse, rédigée par les plùs éminents professeurs et pratieiens dè cette industrie, et nous ne pouvons que souhaiter que la traduction française qui nous en est promise soít publiée le plus rapidement possible.

En résumé le contenu serait impossible.

Il nous suffira d'indiquer les titres de ses diverses parties pour confirmer la valeur de cet important livre rehaussé par une présentation impeccable, aussi bien au point de vue de la qualité du papier que de la valeur et du nombre des tableaux, graphiques et illustrations.

C'est à $M$. O. Langhard, Président de la Commission Suisse du Lait, qu'est due l'idée première de l'ouvrage auquel ont contribué, ainsi que nous 